\title{
Potential effects of reward and loss avoidance in overweight adolescents
}

\author{
Sussanne Reyes ${ }^{1}$, Patricio Peirano' ${ }^{1}$, Beatriz Luna ${ }^{2}$, Betsy Lozoff ${ }^{3}$ and Cecilia Algarín
}

BACKGROUND: Reward system and inhibitory control are brain functions that exert an influence on eating behavior regulation. We studied the differences in inhibitory control and sensitivity to reward and loss avoidance between overweight/ obese and normal-weight adolescents.

METHODS: We assessed 51 overweight/obese and 52 normal-weight 15-y-old Chilean adolescents. The groups were similar regarding sex and intelligence quotient. Using Antisaccade and Incentive tasks, we evaluated inhibitory control and the effect of incentive trials (neutral, loss avoidance, and reward) on generating correct and incorrect responses (latency and error rate).

RESULTS: Compared to normal-weight group participants, overweight/obese adolescents showed shorter latency for incorrect antisaccade responses (186.0 (95\% Cl: 176.8-195.2) vs. $201.3 \mathrm{~ms}(95 \% \mathrm{Cl}: 191.2-211.5), P<0.05)$ and better performance reflected by lower error rate in incentive trials (43.6 (95\% Cl: $37.8-49.4)$ vs. $53.4 \%$ (95\% Cl: $46.8-60.0), P<0.05)$. Overweight/obese adolescents were more accurate on loss avoidance (40.9 (95\% Cl: $33.5-47.7)$ vs. $49.8 \%$ (95\% Cl: $43.0-$ $55.1), P<0.05)$ and reward $(41.0(95 \% \mathrm{Cl}: 34.5-47.5)$ vs. $49.8 \%$ (95\% Cl: 43.0-55.1), $P<0.05$ ) compared to neutral trials.

CONCLUSION: Overweight/obese adolescents showed shorter latency for incorrect responses and greater accuracy in reward and loss avoidance trials. These findings could suggest that an imbalance of inhibition and reward systems influence their eating behavior.

$\mathbf{T}$ here is increasing appreciation of the complex neurobiology of obesity in which cognitive and motivational processes interact in appetitive behaviors. A recent model of obesity proposes that overeating reflects an imbalance between neural circuits related to motivating behavior (reward mechanism) and prepotent response inhibition (1).

Inhibitory control is the ability to voluntarily inhibit dominant, automatic, prepotent, or incompatible responses in favor of a planned response (2). It is an aspect of executive function that is largely mediated by prefrontal cortical function (orbitofrontal cortex and anterior cingulate gyrus) and modulated by the dopaminergic system (3). Inhibitory control is required for suppressing inappropriate/unwanted actions that can interfere with attaining motor, cognitive, or socioemotional goals. It is involved in critical everyday tasks, from preventing impulsive actions to controlling the temptation to overeat (4).

Motivation for action also plays a crucial role in executive behavior. Motivation is influenced by reward processes, with complex brain circuits that involve the orbitofrontal cortex, medial prefrontal cortex, insula, basal ganglia, hippocampus and amygdale, and dopaminergic systems in the midbrain (5). Rewards are stimuli such as objects or events that generate approach to consummatory behavior, learning, positive outcomes, emotions, and hedonic feelings (6). The mesolimbic dopamine reward system plays a key role in giving incentive salience to preferred food (7). Thus, reward-driven decisionmaking may play an important role in overeating behavior (8). For instance, loss of eating control is associated with more body fat gain over time (9) and illicit drug use (10).

Both inhibition and the assessment of potential rewards are crucial to decision-making (11). The model of neural circuit imbalance in obesity indicates that, in vulnerable individuals, a high intake of high-calorie food could alter the ongoing balance between reward and inhibition circuits, resulting in an enhanced reinforcing value of food and a relative weakening of cognitive control modulation. The disrupted balance would thus be a consequence of the resetting of reward thresholds and the weakening of the cortical top-down circuits that regulate inhibition capacity, resulting in impulsive and compulsive food intake (12). There is neuroimaging support for this model. Overweight individuals who were presented with pictures of high-calorie food showed increased neural activation of regions involved in reward circuits compared to normal weight (NW) controls (5).

Our focus is on adolescence, a period characterized by the hypersensitivity to potential rewards and immature cognitive control (13). This combination influences the decision-making process and favors involvement in risk behaviors (11). Only a few studies have examined the association between inhibitory control, reward, and overweight/obesity (OW) in adolescents (14-16). Existing research for this age group suggests that OW

'Sleep and Functional Neurobiology Laboratory, Institute of Nutrition and Food Technology (INTA), University of Chile, Santiago, Chile; ${ }^{2}$ Laboratory of Neurocognitive Development, Western Psychiatric Institute and Clinic, University of Pittsburgh, Pittsburgh, Pennsylvania; ${ }^{3}$ Center for Human Growth and Development, University of Michigan, Ann Arbor, Michigan. Correspondence: Cecilia Algarín (calgarin@inta.uchile.cl) 
Table 1. Background characteristics

\begin{tabular}{|c|c|c|c|c|}
\hline & NW $(n=52)$ & OW $(n=51)$ & $t$ & $P$ value \\
\hline Female (\%) ${ }^{\mathrm{a}}$ & 32.3 & 46.8 & & 0.098 \\
\hline IDA in infancy (\%) & 56.5 & 46.8 & & 0.472 \\
\hline Birth height (cm) & $50.7 \pm 1.9$ & $50.6 \pm 1.8$ & 0.366 & 0.715 \\
\hline Gestational age (weeks) & $39.4 \pm 1.0$ & $39.2 \pm 1.1$ & 0.575 & 0.566 \\
\hline Body weight (kg) & $59.2 \pm 7.2$ & $73.6 \pm 12.6$ & -7.759 & $<0.001$ \\
\hline Body height (cm) & $165.8 \pm 0.1$ & $163.4 \pm 0.1$ & 1.581 & 0.116 \\
\hline Body height (z-score) & $-0.4 \pm 0.4$ & $-0.5 \pm 0.2$ & 0.733 & 0.464 \\
\hline BMI $\left(\mathrm{kg} / \mathrm{m}^{2}\right)$ & $21.4 \pm 1.4$ & $27.4 \pm 3.4$ & -12.724 & $<0.001$ \\
\hline Age of menarche (year) & $12.3 \pm 0.8$ & $11.8 \pm 1.3$ & 1.213 & 0.233 \\
\hline Female Tanner V (\%) & 57.9 & 63.0 & & 0.729 \\
\hline Male Tanner V (\%) & 40.7 & 51.9 & & 0.413 \\
\hline Low SES at 10 years (\%) & 8.1 & 21.0 & & 0.107 \\
\hline Mother self-reported prepregnancy weight (kg) & $66.3 \pm 15.0$ & $71.9 \pm 16.0$ & -1.926 & 0.057 \\
\hline Mother education (y) & $9.7 \pm 2.8$ & $9.3 \pm 2.7$ & 0.778 & 0.438 \\
\hline
\end{tabular}

Values are expressed as means \pm SE. Independent sample T-test.

IDA, iron-deficiency anemia; NW, normal weight; OW, over weight; SES, socioeconomic status.

${ }^{a}$ Chi square test. ${ }^{b}$ Wechsler Intelligence Scale for Children R. Low SES: score < 27.(40)

adolescents had poorer performance on response inhibition, cognitive flexibility, decision-making, and lower sensitivity to reward compared to NW adolescents $(14,15)$. Further, BMI showed a positive association with reward sensitivity in normal weight and overweight subjects that changed to a negative association in the obese group (16). Adolescents may also be particularly sensitive to "motivation" from potential loss (17), but avoidance of loss has received even less attention than response to reward.

OW has become a major public health problem in Chile, with the prevalence in children and adolescents (females $27.1 \%$, males $28.6 \%$ ) among the highest levels in Latin America (18). In the current study, we assessed inhibitory control and sensitivity to reward and loss avoidance in OW and NW Chilean adolescents. We hypothesized that OW adolescents would demonstrate lower inhibitory control and greater sensitivity to reward and loss avoidance than NW adolescents.

\section{RESULTS}

The OW and NW groups were similar in background characteristics in infancy and childhood, although there was a tendency toward higher self-reported prepregnancy maternal weight in the OW compared to NW group (Table 1). By design, groups differed in anthropometric measurements in adolescence.

\section{Latency}

In the Antisaccade task, OW adolescents showed shorter latencies to incorrect saccades than NW adolescents $(F(1,102)=4.9$, $P<0.05$ ) (Table 2). There were no differences in latencies to correct saccades (all $P>0.714$ ). In the Incentive task, there was no significant effect of group on latencies $(P>0.289)$, nor was the interaction between group and trial type significant for latencies $(P>0.231)$.

\section{Accuracy}

Considering all subjects in the Incentive task, there was a significant main effect of trial type $(F(2,172)=4.2, P<0.05)$. Compared to neutral trials, reward trials (44.1 vs. $54.0 \%$, $P<0.01)$ and loss avoidance trials ( 46.6 vs. $54.0 \%, P<0.01$ ) error rates were lower. There was a significant effect of group on error rate $(F(1,86)=4.3, P<0.05)$. Overall, OW participants had a lower error rate than NW participants (Figure 1). This result in OW adolescents was driven by greater accuracy in neutral and loss avoidance trials compared to the NW group (Figure 2). When we analyzed separately the accuracy of each group, we found that OW adolescents showed lower error rate in loss avoidance $(t=-2.4, P<0.01)$ and reward trials $(t=-2.2, P<0.05)$ compared to neutral trials, but accuracy was similar in loss avoidance and reward trials $(t=0.7$, $P=0.709)$ (Figure 2). NW subjects also showed a lower error rate in loss avoidance $(t=-1.5, P=0.05)$ and reward trials 
Table 2. Behavioral results on Antisaccade and Incentive tasks

\begin{tabular}{|c|c|c|}
\hline & NW $(n=52)$ & $\mathrm{OW}(n=51)$ \\
\hline \multicolumn{3}{|l|}{ Antisaccade task ${ }^{\mathrm{a}}$} \\
\hline Error rate & $39.6(33.7-45.6)$ & $41.1(35.7-46.5)$ \\
\hline $\begin{array}{l}\text { Latency correct saccade } \\
\text { (ms) }\end{array}$ & $301.4(282.2-320.7)$ & $299.0(281.6-316.5)$ \\
\hline $\begin{array}{l}\text { Latency incorrect } \\
\text { saccade (ms) }\end{array}$ & $201.3(191.2-211.5)$ & $186.0(176.8-195.2)^{*}$ \\
\hline \multicolumn{3}{|l|}{ Incentive task } \\
\hline \multicolumn{3}{|l|}{ Neutral ${ }^{b}$} \\
\hline $\begin{array}{l}\text { Latency correct } \\
\text { saccade (ms) }\end{array}$ & $414.9(387.5-442.3)$ & $414.7(390.6-438.7)$ \\
\hline $\begin{array}{l}\text { Latency incorrect } \\
\text { saccade (ms) }\end{array}$ & $291.7(278.4-304.9)$ & $298.2(286.5-309.8)$ \\
\hline \multicolumn{3}{|l|}{ Loss avoidance $^{b}$} \\
\hline $\begin{array}{l}\text { Latency correct } \\
\text { saccade (ms) }\end{array}$ & $380.8(355.9-405.7)$ & $392.6(370.8-414.5)$ \\
\hline $\begin{array}{l}\text { Latency incorrect } \\
\text { saccade (ms) }\end{array}$ & $300.4(288.8-312.0)$ & $293.0(282.8-303.2)$ \\
\hline \multicolumn{3}{|l|}{ Reward $^{b}$} \\
\hline $\begin{array}{l}\text { Latency correct } \\
\text { saccade (ms) }\end{array}$ & $415.3(393.8-436.7)$ & $393.6(374.8-412.5)$ \\
\hline $\begin{array}{l}\text { Latency incorrect } \\
\text { saccade (ms) }\end{array}$ & $302.8(286.0-319.5)$ & $293.8(279.1-308.6)$ \\
\hline
\end{tabular}

( $t=-3.1, P<0.01)$ compared to neutral trials. In contrast to the OW group, the NW group error rates differed between loss avoidance and reward trials, being more accurate in the reward trials $(t=-2.0, P<0.05)$ (Figure 2).

\section{DISCUSSION}

This study highlights the association between inhibitory control and OW and contributes to the understanding of the impact of reward and loss avoidance in OW adolescents. Few studies have determined individual differences in inhibition and the modulation of reward and loss avoidance in OW adolescents (14-16,19). We found that OW adolescents had shorter latency for incorrect saccades in inhibitory task and lower error rate in incentive task than NW adolescents.

Short-latency saccades have been associated with higher tendency to make inhibitory errors, suggesting deficits in the ability to inhibit eye-movement responses to distracting stimuli (20). When OW adolescents commit errors, they have shorter latencies suggesting that when inhibition fails it may be due to greater impulsivity (20).

Regarding error rate compared to the NW group, OW adolescents had better accuracy across incentive trials. This greater performance in the OW group could reflect increased sensitivity to incentive trials leading to better performance. It might be possible that OW adolescents were already performing the task at a high level during neutral trials and they were unable to perceive neutral trials as stimuli that did not involve a reward

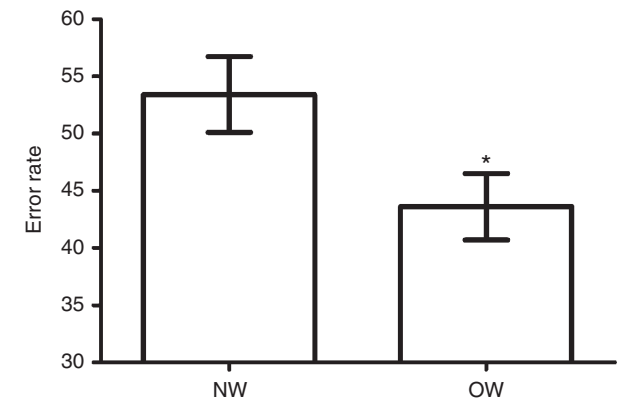

Figure 1. Incentive task error rate in normal-weight (NW) and overweight (OW)/obese groups. Each bar represents the mean values and lines the standard error of the mean. There was a significant difference in error rate between groups. Statistical difference was determined by repeated measures ANOVA. * $P<0.05$.

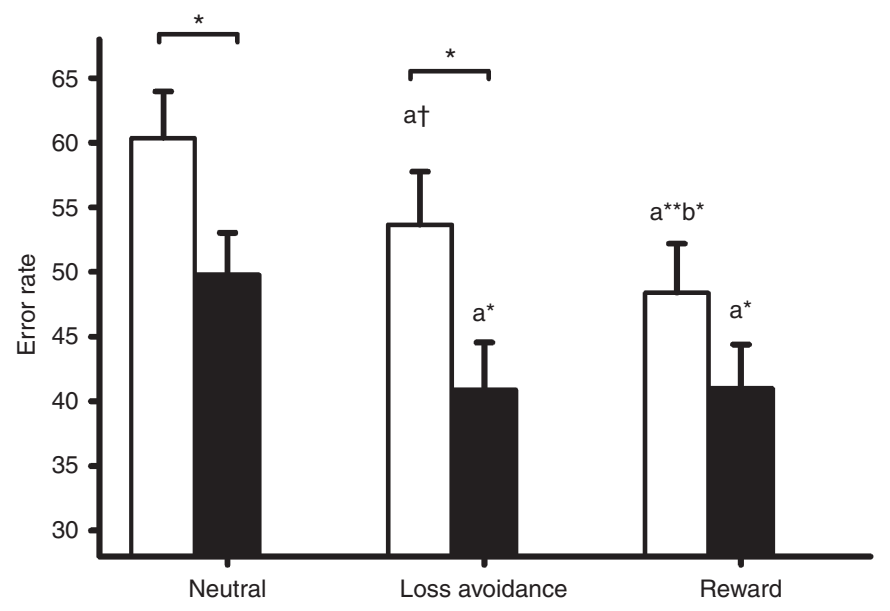

Figure 2. Incentive task error rate for each trial type in normal-weight and overweight/obese groups. Each bar represents the mean values and lines the standard error of the mean. Horizontal lines indicate significant differences between normal-weight (white bars) and overweight/obese (black bars) groups. asignificant difference compared to neutral trial within group. 'bignificant difference compared to loss avoidance trial within group. Statistical differences were determined by repeated measures ANOVA. $+P=0.05 ;{ }^{*} P<0.05 ;{ }^{*} P<0.01$.

value, discerning these trials more "rewarding" (21) and feeling motivated to do it better than NW subjects. While neutral trials did not carry an immediate incentive contingency, they were embedded within an Incentive task that may engage the reward system (21). Studies using this task have shown that adolescents have worse Antisaccade performance than adults (22) but can perform at adult levels when incentives are added (17). Adolescents showed greater activity in striatal function in parallel with increased recruitment of inhibitory control regions during rewarded trials relative to adults, suggesting a specific profile of reward processing in adolescence that may underlie their ability to perform at adult levels when incentives are present $(21,23)$.

Within groups, both OW and NW participants were more efficient in reward and loss avoidance compared to their corresponding neutral trials. However, NW subjects had lower performance in loss avoidance than reward trials, while OW adolescents did not. This may reflect enhanced response for loss avoidance. Such a bias has been related to emotional 
internal conflicts (19), impaired outcome monitoring, inability to learn from experiences, and alterations in motivation (24).

The possible mechanisms underlying the relationship between OW, inhibition, and the modulation of potential rewards and losses are not fully understood. Studies in youth and adults suggest decreased dopamine signaling (receptors and release) in striatal regions, which are linked to reward and habits and also to routines in obesity (25). Diminished striatal dopamine receptors have been associated to altered metabolic activity in both orbitofrontal cortex and anterior cingulate gyrus in obese humans (26). These brain regions contribute to inhibitory control, and dysfunction relates to compulsive behaviors (26) Compared to NW subjects, OW individuals exhibited gray matter volume reductions in the orbitofrontal cortex, postcentral and middle frontal gyrus $(27,28)$. An association between higher BMI and lower metabolic activity in the dorsolateral prefrontal and cingulate brain regions has also been reported (29). Obese subjects showing less activation of reward circuits were at increased risk for weight gain if they carried genetic risk for reduced dopamine signaling (presence of dopamine D2 receptor gene allele). However, an increased activation in these brain regions predicted higher weight gain in subjects who did not have such a genetic risk factor (30). Thus, OW subjects could have differences in brain circuits according to genetic factors that could make them vulnerable to overeating.

Emerging evidence suggests that obesity and addiction share the feature that individuals can express a desire to limit drugs or food consumption but persist despite knowing negative consequences (31). The ability to inhibit the urge to eat desirable food varies among individuals and appears to be a factor that counteracts their vulnerability for overeating (32). Behavioral and neurobiological features described in obese subjects, which are similar to patterns that characterize illicit drugs consumers (33), could implicate neuroadaptations in dopamine signaling as contributors to the disrupted functioning of frontal cortical regions associated with OW (12).

Overall, our data suggest differences in the inhibition process and reward response between OW and NW adolescents. However, we cannot make inferences about causality given the cross-sectional nature of this study. Further, it might be important to use additional measures to determine nutritional status, like body composition or biochemical indices.

In summary, our results provide evidence of weaker inhibitory control and greater motivational sensitivity in OW individuals. They may be particularly sensitive to incentives, affecting decision making and playing an important role in overeating behavior (7). In studying adolescents' behaviors, it is important to understand the neural circuits involved in the way that environmental stimuli influence decisions and actions. Such understanding should help inform strategies and interventions that aim to modify lifestyles toward healthier behaviors. Our findings support the obesity-risk model proposing that overeating reflects an imbalance between circuits inhibiting prepotent responses and circuits of reward (1).

\section{METHODS \\ Subjects}

The study included 103 adolescents who had neurophysiological evaluations in the Sleep and Functional Neurobiology Laboratory, INTA, University of Chile, at 15-16 y of age. All were participants in an ongoing longitudinal study of the behavioral and developmental effects of iron-deficiency anemia in infancy. Detailed descriptions of the population and study design (34) and findings during infancy and childhood have been published elsewhere $(35,36)$.

In brief, inclusion criteria for enrollment at the infancy phase of the study were healthy full-term birth, birth weight $\geq 3.0 \mathrm{~kg}$, without perinatal complications, and absence of acute or chronic illnesses. Infants with iron-deficiency anemia at 6,12 , or 18 mo were considered for neurophysiological evaluations. Randomly chosen infants who were clearly nonanemic (venous $\mathrm{Hb} \geq 115 \mathrm{~g} / \mathrm{l}$ ) also received evaluations. Participants were treated with oral iron for at least 6 mo and had normal hemoglobin concentrations after treatment. No participant had iron-deficiency anemia at subsequent follow-ups.

Parents provided signed informed consent, and adolescents gave their written assent. The original and follow-up protocols were approved and reviewed annually by the Institutional Review Boards of the University of Michigan, Ann Arbor, and Institute of Nutrition and Food Technology (INTA), University of Chile, Santiago.

\section{Materials}

We used the Antisaccade task, which is a refined test of inhibitory control that has been well characterized in animal and human studies (37) and quite widely used in the adolescent research field $(2,17,21,23)$. To examine the effects of incentive types on response inhibition, we also used an Incentive task with potential reward, loss avoidance, and neutral trials (17).

Saccades are rapid eye movements that allow visual stimulus to be foveated and become the new target of attention (17). Eye movements or saccades in these tasks were assessed with an eye-tracking system (Eye-Trac 6; Applied Science Laboratories, Bedford, MA). This system uses a corneal reflection method with bright pupil technology. The point-of-gaze is determined by relating the corneal reflection of an infrared beam, which is projected to the eye to the center of the illuminated pupil rotating with each eye movement. Stimuli were presented using E-Prime software (Psychology Software Tools, Pittsburgh, PA) and displayed on a computer monitor in front of the participant.

\section{Procedure}

Participants were in a darkened room facing the stimulus monitor, seated comfortably $60 \mathrm{~cm}$ away from the monitor center. At the beginning of the experimental session, a 9-point calibration procedure was performed. Before each task, carefully standardized instructions were provided. Testing began after participants demonstrated understanding of procedures.

Antisaccade task. This task probes the ability to exert cognitive control of behavior by exerting voluntary suppression of a prepotent saccadic response. Subjects must inhibit an eye movement towards a visual stimulus and instead make a planned movement to its mirror location. In this task participants were required to fixate a central stimulus for 0.5 to $6.0 \mathrm{~s}$, after which a peripheral target appeared for $1.0 \mathrm{~s}$ at one of four locations randomly presented ( 4 or 8 degrees to the left or right of center fixation). Adolescents were instructed to look in the opposite location of the peripheral target (whose location was unpredictable) as quickly and accurately as possible. Forty-eight experimental trials were presented.

Incentive task. This task explores if the presence of trials with incentives of "reward," "loss avoidance," or "neutral" alters task performance (17). The saccade component is the same as described above, 
but in this task each trial began with 2- or 3-s presentation of one of three possible incentive trials:

(i) Reward trial: A computer image of a bill of one thousand Chilean pesos (1.71 USD) indicated a monetary gain if he/she performed the trial correctly. An incorrect response did not result in "money loss".

(ii) Loss avoidance trial: A torn bill of one thousand Chilean pesos indicated a monetary loss if an error was made. Correct response did not result in "money gain".

(iii) Neutral trial: A green rectangle indicated no incentive, i.e., no money was "gained" or "lost" and the amount of money remained the same, regardless of performance.

Following the fixation cue, indicating that an antisaccade had to be subsequently performed, a peripheral target appeared for $1.0 \mathrm{~s}$ at one of six locations $(9,6$, or 3 degrees to the left or right of the center fixation). Finally, a central stimulus appeared for $1.0 \mathrm{~s}$ to center gaze before the next trial.

The protocol included 20 reward trials, 20 loss avoidance trials, and 20 neutral trials, presented in random order. Participants were encouraged to perform the task as well as they could regardless of incentive trial and as quickly as possible.

The first eye movement with velocity $\geq 30 \%$ s was classified as a correct or incorrect response. Variables of interest included correct saccade (movement made toward the opposite visual field of the peripheral target) and incorrect saccade (movement made toward the peripheral target). Latencies to initiate responses and error rates were also obtained for each trial type (error rate $=$ (number of errors $/$ number of opportunities) ${ }^{\star} 100$ ). Performance on the Incentive task was evaluated comparing error rate and latencies by group and trial type. The effect of reward and loss avoidance trials compared to neutral trials in each group was interpreted as the modulation of reward and loss avoidance in inhibitory control.

\section{Eye-Tracking Data Processing}

Eye movement data were scored off-line using ILAB software (Northwestern University Medical School and V. A. Healthcare System, Chicago, IL) (38) and MATLAB (MathWorks, Natick, MA), which calculated the direction, latency, and accuracy of saccades. Eye movement latencies $<70 \mathrm{~ms}$ were excluded in the analysis. Before final classification of saccades, performance on each trial was checked to identify blink artifacts and occasional failures of the software that detected saccades.

\section{Anthropometric Measures}

Trained personnel made weight and height measurements using standardized techniques (without shoes, wearing underwear, and in the Frankfurt position) on the same machine calibrated every day. Weight to the closest $0.1 \mathrm{~kg}$ and height to the closest $0.1 \mathrm{~cm}$ were measured using a SECA scale (model 700, Seca, Hamburg, Germany). BMI was calculated for each participant as the ratio of weight in kilograms divided by the square of height in meters. Sex-and age-specific BMI percentiles and $\mathrm{z}$-scores were calculated and categorized according to the cut-off points recommended by the World Health Organization (39) as NW (BMI z-score $\geq-2$ to $<1$ ) and OW (BMI z-score $\geq 1$, i.e., overweight and obese).

\section{Data Analysis}

We used multivariate ANOVA to assess the relationship between group (NW and OW) and performance (error rate and latencies) on the Antisaccade task. We conducted repeated measures ANOVA to examine the main effects and interaction of performance on the Incentive task and group (NW and OW). The withinparticipants factor was trial type (reward, loss avoidance, and neutral), and the between-participants factor was group. Post-hoc paired $t$-tests were conducted using Bonferroni correction for multiple comparisons. The model included sex, iron status in infancy, and socioeconomic status as covariates. Statistical analyses were conducted with SPSS software version 19.0 (SPSS, Chicago, IL). All significance tests were two-tailed; a $P$ value $<0.05$ was considered statistically significant.

\section{ACKNOWLEDGMENTS}

We would like to express our gratitude to the adolescents and parents whose participation made this study possible. We also thank the technicians of the Sleep and Functional Neurobiology Laboratory of INTA, University of Chile, who contributed during the course of this study, and drivers for providing careful transportation services to participants.

\section{STATEMENT OF FINANCIAL SUPPORT}

This study was supported by Chilean National Fund for Scientific and Technological Development grant 1110513, and US National Institutes of Health (Bethesda, MD) grant HD33487.

Disclosures: All authors have nothing to disclose.

\section{REFERENCES}

1. Volkow ND, Wang GJ, Fowler JS, Telang F. Overlapping neuronal circuits in addiction and obesity: evidence of systems pathology. Philos Trans R Soc Lond, B, Biol Sci 2008;363:3191-200.

2. Ordaz SJ, Foran W, Velanova K, Luna B. Longitudinal growth curves of brain function underlying inhibitory control through adolescence. J Neurosci 2013;33:18109-24.

3. Logue SF, Gould TJ. The neural and genetic basis of executive function: attention, cognitive flexibility, and response inhibition. Pharmacol Biochem Behav 2014;123:45-54.

4. Mostofsky SH, Simmonds DJ. Response inhibition and response selection: two sides of the same coin. J Cogn Neurosci 2008;20:751-61.

5. Stoeckel LE, Kim J, Weller RE, Cox JE, Cook EW 3rd, Horwitz B. Effective connectivity of a reward network in obese women. Brain Res Bull 2009;79:388-95.

6. Schultz W. Dopamine signals for reward value and risk: basic and recent data. Behav Brain Funct 2010;6:24.

7. Berridge KC, Ho CY, Richard JM, DiFeliceantonio AG. The tempted brain eats: pleasure and desire circuits in obesity and eating disorders. Brain Res 2010;1350:43-64.

8. Pignatti R, Bertella L, Albani G, Mauro A, Molinari E, Semenza C. Decision-making in obesity: a study using the Gambling Task. Eat Weight Disord 2006;11:126-32.

9. Tanofsky-Kraff M, Cohen ML, Yanovski SZ, et al. A prospective study of psychological predictors of body fat gain among children at high risk for adult obesity. Pediatrics 2006;117:1203-9.

10. Ross HE, Ivis F. Binge eating and substance use among male and female adolescents. Int J Eat Disord 1999;26:245-60.

11. Blakemore SJ, Robbins TW. Decision-making in the adolescent brain. Nat Neurosci 2012;15:1184-91.

12. Volkow ND, Wang GJ, Baler RD. Reward, dopamine and the control of food intake: implications for obesity. Trends Cogn Sci (Regul Ed) 2011;15:37-46.

13. Naneix F, Marchand AR, Pichon A, Pape JR, Coutureau E. Adolescent stimulation of $\mathrm{D} 2$ receptors alters the maturation of dopamine-dependent goal-directed behavior. Neuropsychopharmacology 2013;3:1-9.

14. Verdejo-García A, Pérez-Expósito M, Schmidt-Río-Valle J, et al. Selective alterations within executive functions in adolescents with excess weight. Obesity (Silver Spring) 2010;18:1572-8.

15. Delgado-Rico E, Río-Valle JS, González-Jiménez E, Campoy C, VerdejoGarcía A. BMI predicts emotion-driven impulsivity and cognitive inflexibility in adolescents with excess weight. Obesity (Silver Spring) 2012;20:1604-10.

16. Verbeken S, Braet C, Lammertyn J, Goossens L, Moens E. How is reward sensitivity related to bodyweight in children? Appetite 2012;58:478-83.

17. Geier CF, Luna B. Developmental effects of incentives on response inhibition. Child Dev 2012;83:1262-74.

18. Rodríguez L. Situación nutricional del escolar y adolescente en Chile. Rev Chil Pediatr 2007;78:523-33.

19. Matton A, Goossens L, Braet C, Vervaet M. Punishment and reward sensitivity: are naturally occurring clusters in these traits related to eating and weight problems in adolescents? Eur Eat Disord Rev 2013;21:184-94.

20. Luna B, Velanova K, Geier CF. Development of eye-movement control. Brain Cogn 2008;68:293-308. 


\section{Reward, loss avoidance, and overweight}

21. Geier CF, Terwilliger R, Teslovich T, Velanova K, Luna B. Immaturities in reward processing and its influence on inhibitory control in adolescence. Cereb Cortex 2010;20:1613-29.

22. Luna B. Developmental changes in cognitive control through adolescence. Adv Child Dev Behav 2009;37:233-78.

23. Padmanabhan A, Geier CF, Ordaz SJ, Teslovich T, Luna B. Developmental changes in brain function underlying the influence of reward processing on inhibitory control. Dev Cogn Neurosci 2011;1:517-29.

24. Bischoff-Grethe A, McCurdy D, Grenesko-Stevens E, et al. Altered brain response to reward and punishment in adolescents with Anorexia nervosa. Psychiatry Res 2013;214:331-40.

25. Wang GJ, Volkow ND, Logan J, et al. Brain dopamine and obesity. Lancet 2001;357:354-7.

26. Volkow ND, Wang GJ, Telang F, et al. Low dopamine striatal D2 receptors are associated with prefrontal metabolism in obese subjects: possible contributing factors. Neuroimage 2008;42:1537-43.

27. Yokum S, Ng J, Stice E. Relation of regional gray and white matter volumes to current BMI and future increases in BMI: a prospective MRI study. Int J Obes (Lond) 2012;36:656-64.

28. Maayan L, Hoogendoorn C, Sweat V, Convit A. Disinhibited eating in obese adolescents is associated with orbitofrontal volume reductions and executive dysfunction. Obesity (Silver Spring) 2011;19:1382-7.

29. Volkow ND, Wang GJ, Telang F, et al. Inverse association between BMI and prefrontal metabolic activity in healthy adults. Obesity (Silver Spring) 2009;17:60-5.

30. Stice E, Yokum S, Bohon C, Marti N, Smolen A. Reward circuitry responsivity to food predicts future increases in body mass: moderating effects of DRD2 and DRD4. Neuroimage 2010;50:1618-25.
31. Fandiño J, Moreira RO, Preissler C, et al. Impact of binge eating disorder in the psychopathological profile of obese women. Compr Psychiatry 2010;51:110-4.

32. Wang GJ, Volkow ND, Telang F, et al. Evidence of gender differences in the ability to inhibit brain activation elicited by food stimulation. Proc Natl Acad Sci USA 2009;106:1249-54.

33. Lillis J, Levin ME, Trafton JA. Elevated BMI and illicit drug use are associated with decreased ability to inhibit prepotent behaviors. Addict Behav 2012;37:544-7.

34. Lozoff B, De Andraca I, Castillo M, Smith JB, Walter T, Pino P. Behavioral and developmental effects of preventing iron-deficiency anemia in healthy full-term infants. Pediatrics 2003;112:846-54.

35. Peirano P, Algarin C, Chamorro R, Manconi M, Lozoff B, Ferri R. Iron deficiency anemia in infancy exerts long-term effects on the tibialis anterior motor activity during sleep in childhood. Sleep Med 2012;13: 1006-12.

36. Algarín C, Nelson CA, Peirano P, Westerlund A, Reyes S, Lozoff B. Irondeficiency anemia in infancy and poorer cognitive inhibitory control at age 10 years. Dev Med Child Neurol 2013;55:453-8.

37. Munoz DP, Everling S. Look away: the anti-saccade task and the voluntary control of eye movement. Nat Rev Neurosci 2004;5:218-28.

38. Gitelman DR. ILAB: a program for postexperimental eye movement analysis. Behav Res Methods Instrum Comput 2002;34:605-12.

39. World Health Organization. Growth reference 5-19 years, 2007. http:// www.who.int/growthref/who2007_bmi_for_age/en/.

40. Kang Sim DE, Cappiello M, Castillo M, et al. Postnatal Growth Patterns in a Chilean Cohort: The Role of SES and Family Environment. Int J Pediatr 2012;2012:354060. 